future functional experiments to further characterise and elucidate B. cepacia infection.

http://dx.doi.org/10.1016/j.ijid.2012.05.840

Type: Poster Presentation

Final Abstract Number: 45.050?Session: Bacterial Infections?Date: Friday, June 15, 2012?Time: 12:45-14:15?Room: Poster \& Exhibition Area

\section{Study of detection Vibrio Cholerae 01 from Karoon river waters and their role in the public health}

M. Mehdinejad ${ }^{1}$,, N. NassirAbadi ${ }^{1}$, H. Godarzi ${ }^{2}$

${ }^{1}$ Ahvaz Jundishapur University of Medical Sciences (AJUMS), Ahvaz, Iran, Islamic Republic of

${ }^{2}$ Ahvaz Jundishapur University of Medical Sciences, Ahvaz, Iran, Islamic Republic of

Background: The watershed of Dez and Karoon rivers located in middle Zagrous mountain with area about 68481-km, thus watershed is a part of Persian gulf watershed. Cholera, an acute intestinal infection caused by the bacterium Vibrio cholerae, is a historically feared epidemic diarrheal disease that remains a major public health problem in many parts of Africa, Asia, and Latin America. Vibrio cholerae $\mathrm{O} 1$ exists as two major serotypes, Inaba and Ogawa, a member of the family Vibrionaceae. Vibrio cholerae is transmitted through fresh water contaminated with fecal matter. Foodborne infections have been traced to raw or inadequately cooked shellfish and other seafood. The target of study the segregate Vibrio cholerae 01 (Vibrionaceae) in the Karun Ahvaz river.

Methods: In four stages (April, May, June and July 2010), a total 100 samples of water from Karoon River Ahvaz were collected. During the study period the recorded river temperature was about 25-28 ( $\mathrm{C}$ and $\mathrm{pH}$ ranged from 7 to 8 . Swabs were cultured onto thiosulphate citrate bile sucrose and MacConkey, and morphological colonies compatible with Vibrio were characterized by oxidase test and agglutinated with antiserum (Difco, Detroit, MI, USA) for serotype determination. Also V. cholerae biochemical tests with API 20E (BioMerieux, Marcy-l'Etoile, France).

Results: From 100 samples of water Karoon River in Ahvaz, Iran, $8(8 \%)$ sample were positive for Vibrio cholerae strains. The isolated strains from water Karoon River in Ahvaz, Iran, were Vibrio cholerae $\mathrm{O} 1$ (inaba).

Conclusion: The priorities for cholera control remain public health interventions through improved water and sanitation, improved surveillance and access to health care facilities, and further development of appropriate vaccines.

http://dx.doi.org/10.1016/j.ijid.2012.05.841

\section{Type: Poster Presentation}

Final Abstract Number: 45.051?Session: Bacterial Infections?Date: Friday, June 15, 2012?Time: 12:45-14:15?Room: Poster \& Exhibition Area

\section{Safety of polymyxin-B- based hemoperfusion in kidney and liver transplant recipients}

V. Morabito*, G. Ferretti, F. Pugliese, S. Novelli, G. Novelli

Sapienza University, Rome, Italy

Background: Infection represents one of the primary barriers to successful organ transplantation and an early diagnosis represent the goal of therapy. Our principal end point was to use a new assay Test EAA which has been developed to rapidly detect endotoxin acivity. Furthermore we aim to prove the validity and safety of removal of endotoxins using Polymyxin-B based hemoperfusion (PMX-DHP).

Methods: The criteria for inclusion in the study were the following findings: infection was suspected when patients had at least 2 of the 4 criteria of systemic inflammatory response syndrome (SIRS). Following these criteria, the Test EAA was performed on 71 patients (29 liver transplant and 42 kidney transplant). Twenty eight patients $(39,5 \%)$ with $E A>0.60$ were enrolled in this study and received treatment to remove endotoxins (PMX-DHP). Each treatment was performed for two hours with a blood flow rate of $100 \mathrm{~mL} / \mathrm{min}$. All the patients were treated with PMX-DHP until an $\mathrm{EA}<0.4$ was found.

Results: No relevant adverse events were observed during the 72 treatments performed.Before performing PMX-DHP treatments, the median EA was 0.81(range 0.62-1.25) and 0.73(range 0.610.98 ) in liver and kidney transplant patients respectively. In liver transplant patients two PMX-DHP treatments were performed on 7 patients [median EA $=0.69(0.62-0.76)$ ], three treatments on 4 patients[median EA $=0.84(0.77-0.91)]$ and four treatment on 3 patients [median EA $=1.11(0.95-1.25)$ ]. At the end of the endotoxin removal therapy, the median EA level was 0.33[0.22-0.4].The stabilization of hemodynamic and inflammatory frameworks were observed after the PMX-DHP.At 30 days of follow up all patients were alive with a good graft function and low level of endotoxin activity.

Conclusion: Given the progress achieved and considering the particular difficulties in the diagnosis of transplant patients, we think that might be useful to determine the endotoxin activity routinely in these patients. Accordingly, larger multicenter clinical trials will be necessary to accurately assess the benefits of EA essay plus DHP-PMX for transplant patients with endotoxemia and suspect of infection.

http://dx.doi.org/10.1016/j.ijid.2012.05.842 